\title{
Basins of attraction of certain rational anti-competitive system of difference equations in the plane
}

\author{
Samra Moranjkić and Zehra Nurkanović*
}

\section{"Correspondence:}

zehra.nurkanovic@untz.ba

Department of Mathematics,

University of Tuzla, Tuzla, Bosnia and

Herzegovina

\begin{abstract}
We investigate the global asymptotic behavior of solutions of the following anti-competitive system of rational difference equations:

$$
x_{n+1}=\frac{\gamma_{1} y_{n}}{A_{1}+x_{n}}, \quad y_{n+1}=\frac{\beta_{2} x_{n}}{A_{2}+B_{2} x_{n}+y_{n}}, \quad n=0,1, \ldots
$$

where the parameters $\gamma_{1}, \beta_{2}, A_{1}, A_{2}$ and $B_{2}$ are positive numbers and the initial conditions $\left(x_{0}, y_{0}\right)$ are arbitrary nonnegative numbers. We find the basins of attraction of all attractors of this system, which are the equilibrium points and period-two solutions.
\end{abstract}

MSC: 39A10; 39A11

Keywords: difference equations; anti-competitive; map; stability; stable manifold

\section{Introduction}

A first-order system of difference equations

$$
\left\{\begin{array}{l}
x_{n+1}=f\left(x_{n}, y_{n}\right), \\
y_{n+1}=g\left(x_{n}, y_{n}\right),
\end{array} \quad n=0,1, \ldots,\left(x_{0}, y_{0}\right) \in \mathcal{R},\right.
$$

where $\mathcal{R} \subset \mathbb{R}^{2},(f, g): \mathcal{R} \rightarrow \mathcal{R}, f, g$ are continuous functions is competitive if $f(x, y)$ is non-decreasing in $x$ and non-increasing in $y$, and $g(x, y)$ is non-increasing in $x$ and nondecreasing in $y$.

System (1) where the functions $f$ and $g$ have a monotonic character opposite of the monotonic character in competitive system will be called anti-competitive.

We consider the following anti-competitive system of difference equations:

$$
x_{n+1}=\frac{\gamma_{1} y_{n}}{A_{1}+x_{n}}, \quad y_{n+1}=\frac{\beta_{2} x_{n}}{A_{2}+B_{2} x_{n}+y_{n}}, \quad n=0,1, \ldots
$$

where the parameters $A_{1}, \gamma_{1}, A_{2}, B_{2}$ and $\beta_{2}$ are positive numbers and the initial conditions $\left(x_{0}, y_{0}\right)$ are arbitrary nonnegative numbers. In the classification of all linear fractional systems in [1], System (2) was mentioned as System $(16,39)$.

○ 2012 Moranjkić and Nurkanović; licensee Springer. This is an Open Access article distributed under the terms of the Creative Commons Attribution License (http://creativecommons.org/licenses/by/2.0), which permits unrestricted use, distribution, and reproduction in any medium, provided the original work is properly cited. 
Competitive and cooperative systems of the form (1) were studied by many authors such as Clark and Kulenović [2], Clark, Kulenović and Selgrade [3], Hirsch and Smith [4], Kulenović and Ladas [5], Kulenović and Merino [6], Kulenović and Nurkanović [7, 8], GarićDemirović, Kulenović and Nurkanović $[9,10]$, Smith [11, 12] and others.

The study of anti-competitive systems started in [13] and has advanced since then (see $[14,15])$. The principal tool of the study of anti-competitive systems is the fact that the second iterate of the map associated with an anti-competitive system is a competitive map, and so the elaborate theory for such maps developed recently in $[4,16,17]$ can be applied.

The main result on the global behavior of System (2) is the following theorem.

\section{Theorem 1}

(a) If $\beta_{2} \gamma_{1} \leq A_{1} A_{2}$, then $E_{0}=(0,0)$ is a unique equilibrium, and the basin of attraction of this equilibrium is $\mathcal{B}\left(E_{0}\right)=\{(x, y): x \geq 0, y \geq 0\}$ (see Figure $1(a)$ ).

(b) If $\beta_{2} \gamma_{1}-A_{1} A_{2}>-B_{2}\left[A_{1}^{2}+\gamma_{1}\left(A_{2}-A_{1} B_{2}\right)\right]$ and $\beta_{2} \gamma_{1}-A_{1} A_{2}>0$, then there exist two equilibrium points: $E_{0}$ which is a repeller and $E_{+}$which is an interior saddle point, and minimal period-two solutions $A_{0}=\left(0, \frac{\beta_{2} \gamma_{1}-A_{1} A_{2}}{\gamma_{1} B_{2}}\right)$ and $B_{0}=\left(\frac{\beta_{2} \gamma_{1}-A_{1} A_{2}}{A_{1} B_{2}}, 0\right)$ which are locally asymptotically stable. There exists a set $\mathcal{C} \subset \mathcal{R}=[0, \infty) \times[0, \infty)$ such that $E_{0} \in \mathcal{C}$, and $\mathcal{W}^{s}\left(E_{+}\right)=\mathcal{C} \backslash E_{0}$ is an invariant subset of the basin of attraction of $E_{+}$. The $\operatorname{set} \mathcal{C}$ is a graph of a strictly increasing continuous function of the first variable on an interval and separates $\mathcal{R}$ into two connected and invariant components, namely

$$
\mathcal{W}_{-}:=\left\{\mathbf{x} \in \mathcal{R} \backslash \mathcal{C}: \exists \mathbf{x}^{\prime} \in \mathcal{C} \text { with } \mathbf{x} \preceq_{\text {se }} \mathbf{x}^{\prime}\right\}, \quad \mathcal{W}_{+}:=\left\{\mathbf{x} \in \mathcal{R} \backslash \mathcal{C}: \exists \mathbf{x}^{\prime} \in \mathcal{C} \text { with } \mathbf{x}^{\prime} \preceq_{s e} \mathbf{x}\right\},
$$

which satisfy (see Figure 1(b)):

(i) If $\left(x_{0}, y_{0}\right) \in \mathcal{W}_{+}$, then

$$
\lim _{n \rightarrow \infty}\left(x_{2 n}, y_{2 n}\right)=\left(\frac{\beta_{2} \gamma_{1}-A_{1} A_{2}}{A_{1} B_{2}}, 0\right)=B_{0}
$$

and

$$
\lim _{n \rightarrow \infty}\left(x_{2 n+1}, y_{2 n+1}\right)=\left(0, \frac{\beta_{2} \gamma_{1}-A_{1} A_{2}}{\gamma_{1} B_{2}}\right)=A_{0} .
$$

(ii) If $\left(x_{0}, y_{0}\right) \in \mathcal{W}_{-}$, then

$$
\lim _{n \rightarrow \infty}\left(x_{2 n}, y_{2 n}\right)=\left(0, \frac{\beta_{2} \gamma_{1}-A_{1} A_{2}}{\gamma_{1} B_{2}}\right)=A_{0}
$$

and

$$
\lim _{n \rightarrow \infty}\left(x_{2 n+1}, y_{2 n+1}\right)=\left(\frac{\beta_{2} \gamma_{1}-A_{1} A_{2}}{A_{1} B_{2}}, 0\right)=B_{0} .
$$

(c) If $0<\beta_{2} \gamma_{1}-A_{1} A_{2}=-B_{2}\left[A_{1}^{2}+\gamma_{1}\left(A_{2}-A_{1} B_{2}\right)\right]$, then (see Figure 1(c))

(i) There exist two equilibrium points: $E_{0}$ which is a repeller and $E_{+} \in \operatorname{int}(\mathcal{R})$ which is a non-hyperbolic, and an infinite number of minimal period-two solutions

$$
A_{x}=\left(x, \frac{\beta_{2} \gamma_{1}-A_{1} A_{2}-x A_{1} B_{2}}{\gamma_{1} B_{2}}\right),
$$




$$
B_{x}=\left(\frac{\beta_{2} \gamma_{1}-A_{1} A_{2}-x A_{1} B_{2}}{B_{2}\left(x+A_{1}\right)}, \frac{-x \beta_{2} \gamma_{1}}{\left(A_{1}-\gamma_{1} B_{2}\right)\left(x+A_{1}\right)}\right)
$$

for $x \in\left[0, \frac{\beta_{2} \gamma_{1}-A_{1} A_{2}}{A_{1} B_{2}}\right]$, that belong to the segment of the line (15) in the first quadrant.

(ii) All minimal period-two solutions and the equilibrium $E_{+}$are stable but not asymptotically stable.

(iii) There exists a family of strictly increasing curves $\mathcal{C}_{+}, \mathcal{C}_{A_{x}}, \mathcal{C}_{B_{x}}$ for $x \in\left(0, \frac{\beta_{2} \gamma_{1}-A_{1} A_{2}}{A_{1} B_{2}}\right)$ and

$$
\mathcal{C}_{A_{0}}=\{(x, y): x=0, y>0\}, \quad \mathcal{C}_{B_{0}}=\{(x, y): x>0, y=0\}
$$

that emanate from $E_{0}$ and $A_{x} \in \mathcal{C}_{A_{x}}, B_{x} \in \mathcal{C}_{B_{x}}$ for all $x \in\left[0, \frac{\beta_{2} \gamma_{1}-A_{1} A_{2}}{A_{1} B_{2}}\right)$, such that the curves are pairwise disjoint, the union of all the curves equals $\mathbb{R}_{+}^{2}$. Solutions with initial points in $\mathcal{C}_{+}$converge to $E_{+}$and solutions with an initial point in $\mathcal{C}_{A_{x}}$ have even-indexed terms converging to $A_{x}$ and odd-indexed terms converging to $B_{x}$; solutions with an initial point in $\mathcal{C}_{B_{x}}$ have even-indexed terms converging to $B_{x}$ and odd-indexed terms converging to $A_{x}$.

(d) If $0<\beta_{2} \gamma_{1}-A_{1} A_{2}<-B_{2}\left[A_{1}^{2}+\gamma_{1}\left(A_{2}-A_{1} B_{2}\right)\right]$, then System (2) has two equilibrium points: $E_{0}$ which is a repeller and $E_{+}$which is locally asymptotically stable, and minimal period-two solutions $A_{0}$ and $B_{0}$ which are saddle points. The basin of attraction of the equilibrium point $E_{+}$is the set

$$
\mathcal{B}\left(E_{+}\right)=\{(x, y): x>0, y>0\}
$$

and solutions with an initial point in $\{(x, y): x=0, y>0\}$ have even-indexed terms converging to $A_{0}$ and odd-indexed terms converging to $B_{0}$, solutions with an initial point in $\{(x, y): x>0, y=0\}$ have even-indexed terms converging to $B_{0}$ and odd-indexed terms converging to $A_{0}$ (see Figure 1(d)).

\section{Preliminaries}

We now give some basic notions about systems and maps in the plane of the form (1).

Consider a map $T=(f, g)$ on a set $\mathcal{R} \subset \mathbb{R}^{2}$, and let $E \in \mathcal{R}$. The point $E \in \mathcal{R}$ is called a fixed point if $T(E)=E$. An isolated fixed point is a fixed point that has a neighborhood with no other fixed points in it. A fixed point $E \in \mathcal{R}$ is an attractor if there exists a neighborhood $\mathcal{U}$ of $E$ such that $T^{n}(\mathbf{x}) \rightarrow E$ as $n \rightarrow \infty$ for $\mathbf{x} \in \mathcal{U}$; the basin of attraction is the set of all $\mathbf{x} \in \mathcal{R}$ such that $T^{n}(\mathbf{x}) \rightarrow E$ as $n \rightarrow \infty$. A fixed point $E$ is a global attractor on a set $\mathcal{K}$ if $E$ is an attractor and $\mathcal{K}$ is a subset of the basin of attraction of $E$. If $T$ is differentiable at a fixed point $E$, and if the Jacobian $J_{T}(E)$ has one eigenvalue with modulus less than one and a second eigenvalue with modulus greater than one, $E$ is said to be a saddle. See [18] for additional definitions.

Here we give some basic facts about the monotone maps in the plane, see $[11,16,17,19]$. Now, we write System (2) in the form

$$
\left(\begin{array}{l}
x \\
y
\end{array}\right)_{n+1}=T\left(\begin{array}{l}
x \\
y
\end{array}\right)_{n}
$$




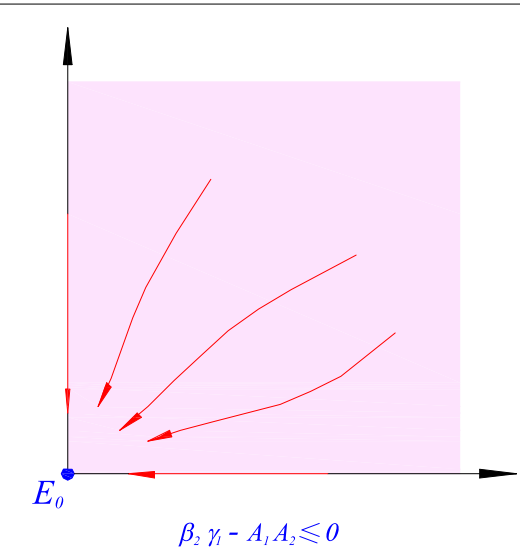

(a)

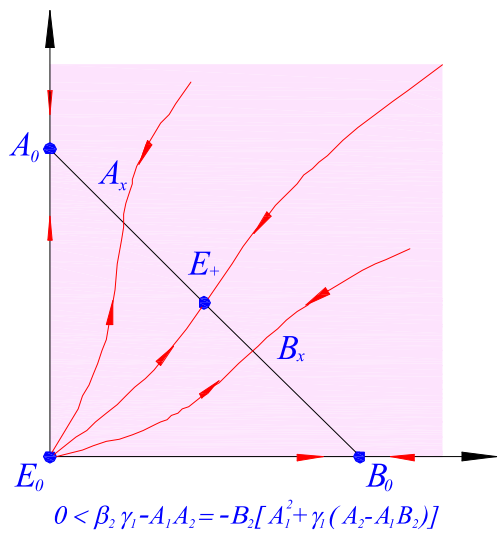

(c)

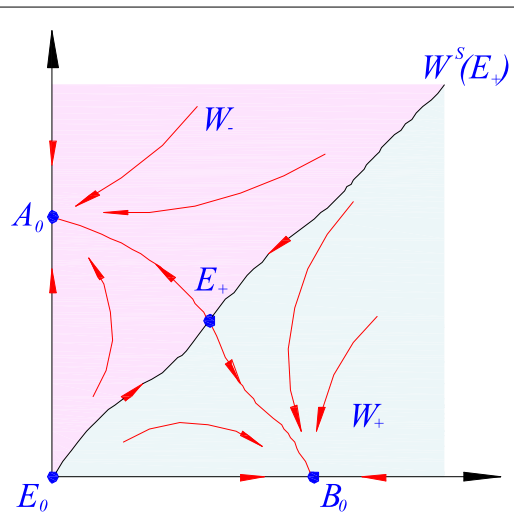

$\beta_{2} \gamma_{1}-A_{1} A_{2}>0$

$\beta_{2} \gamma_{1}-A_{1} A_{2}>-B_{2}\left[A_{1}^{2}+\gamma_{1}\left(A_{2}-A_{1} B_{2}\right)\right]$

(b)

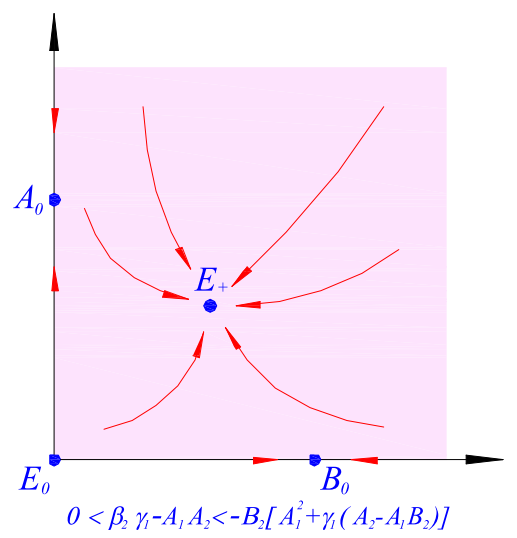

(d)

Figure 1 Basins of attraction

where the map $T$ is given as

$$
T:\left(\begin{array}{l}
x \\
y
\end{array}\right) \rightarrow\left(\begin{array}{c}
\frac{\gamma_{1} y}{A_{1}+x} \\
\frac{\beta_{2} x}{A_{2}+B_{2} x+y}
\end{array}\right)=\left(\begin{array}{l}
f(x, y) \\
g(x, y)
\end{array}\right) .
$$

The map $T$ may be viewed as a monotone map if we define a partial order on $\mathbb{R}^{2}$ so that the positive cone in this new partial order is the fourth quadrant. Specifically, for $\mathbf{v}=\left(v_{1}, v_{2}\right)$, $\mathbf{w}=\left(w_{1}, w_{2}\right) \in \mathbb{R}^{2}$ we say that $\mathbf{v} \preceq \mathbf{w}$ if $v_{1} \leq w_{1}$ and $w_{2} \leq v_{2}$. Two points $\mathbf{v}, \mathbf{w} \in \mathbb{R}_{+}^{2}$ are said to be related if $\mathbf{v} \preceq \mathbf{w}$ or $\mathbf{w} \preceq \mathbf{v}$. Also, a strict inequality between points may be defined as $\mathbf{v} \prec \mathbf{w}$ if $\mathbf{v} \preceq \mathbf{w}$ and $\mathbf{v} \neq \mathbf{w}$. A stronger inequality may be defined as $\mathbf{v} \prec \prec \mathbf{w}$ if $v_{1}<w_{1}$ and $w_{2}<v_{2}$. A map $f: \operatorname{int} \mathbb{R}_{+}^{2} \rightarrow \operatorname{Int} \mathbb{R}_{+}^{2}$ is strongly monotone if $\mathbf{v} \prec \mathbf{w}$ implies that $f(\mathbf{v}) \prec \prec$ $f(\mathbf{w})$ for all $\mathbf{v}, \mathbf{w} \in \operatorname{Int} \mathbb{R}_{+}^{2}$. Clearly, being related is an invariant under iteration of a strongly monotone map. Differentiable strongly monotone maps have Jacobian with constant sign 
configuration

$$
\left[\begin{array}{ll}
+ & - \\
- & +
\end{array}\right]
$$

The mean value theorem and the convexity of $\mathbb{R}_{+}^{2}$ may be used to show that $T$ is monotone, as in [20].

For $\mathbf{x}=\left(x_{1}, x_{2}\right) \in \mathbb{R}^{2}$, define $Q_{l}(\mathbf{x})$ for $l=1, \ldots, 4$ to be the usual four quadrants based at $\mathbf{x}$ and numbered in a counterclockwise direction, for example, $Q_{1}(\mathbf{x})=\left\{\mathbf{y}=\left(y_{1}, y_{2}\right) \in \mathbb{R}^{2}\right.$ : $\left.x_{1} \leq y_{1}, x_{2} \leq y_{2}\right\}$.

The following definition is from [11].

Definition 1 Let $\mathcal{S}$ be a nonempty subset of $\mathbb{R}^{2}$. A competitive map $T: \mathcal{S} \rightarrow \mathcal{S}$ is said to satisfy condition $(O+)$ if for every $x, y$ in $\mathcal{S}, T(x) \preceq_{n e} T(y)$ implies $x \preceq_{n e} y$, and $T$ is said to satisfy condition (O-) if for every $x, y$ in $\mathcal{S}, T(x) \preceq_{n e} T(y)$ implies $y \preceq_{n e} x$.

The following theorem was proved by de Mottoni-Schiaffino for the Poincaré map of a periodic competitive Lotka-Volterra system of differential equations. Smith generalized the proof to competitive and cooperative maps [11].

Theorem 2 Let $\mathcal{S}$ be a nonempty subset of $\mathbb{R}^{2}$. If $T$ is a competitive map for which (O+) holds then for all $x \in \mathcal{S},\left\{T^{n}(x)\right\}$ is eventually componentwise monotone. If the orbit of $x$ has compact closure, then it converges to a fixed point of $T$. If instead (O-) holds, then for all $x \in \mathcal{S},\left\{T^{2 n}\right\}$ is eventually componentwise monotone. If the orbit of $x$ has compact closure in $\mathcal{S}$, then its omega limit set is either a period-two orbit or a fixed point.

The following result is from [11], with the domain of the map specialized to be the Cartesian product of intervals of real numbers. It gives a sufficient condition for conditions $(\mathrm{O}+)$ and $(O-)$.

Theorem 3 Let $\mathcal{R} \subset \mathbb{R}^{2}$ be the Cartesian product of two intervals in $\mathbb{R}$. Let $T: \mathcal{R} \rightarrow \mathcal{R}$ be a $C^{1}$ competitive map. If $T$ is injective and $\operatorname{det} J_{T}(x)>0$ for all $x \in \mathcal{R}$ then $T$ satisfies $(O+)$. If $T$ is injective and $\operatorname{det} J_{T}(x)<0$ for all $x \in \mathcal{R}$ then $T$ satisfies $(O-)$.

Next two results are from $[17,21]$.

Theorem 4 Let $T$ be a competitive map on a rectangular region $\mathcal{R} \subset \mathbb{R}^{2}$. Let $\overline{\mathbf{x}} \in \mathcal{R}$ be a fixed point of $T$ such that $\Delta:=\mathcal{R} \cap \operatorname{int}\left(Q_{1}(\overline{\mathbf{x}}) \cup Q_{3}(\overline{\mathbf{x}})\right)$ is nonempty (i.e., $\overline{\mathbf{x}}$ is not the $N W$ or $S E$ vertex of $\mathcal{R}$ ), and $T$ is strongly competitive on $\Delta$. Suppose that the following statements are true.

a. The map $T$ has a $C^{1}$ extension to a neighborhood of $\overline{\mathbf{x}}$.

b. The Jacobian matrix of $T$ at $\overline{\mathbf{x}}$ has real eigenvalues $\lambda$, $\mu$ such that $0<|\lambda|<\mu$, where

$|\lambda|<1$, and the eigenspace $E^{\lambda}$ associated with $\lambda$ is not a coordinate axis.

Then there exists a curve $\mathcal{C} \subset \mathcal{R}$ through $\overline{\mathbf{x}}$ that is invariant and a subset of the basin of attraction of $\overline{\mathbf{x}}$, such that $\mathcal{C}$ is tangential to the eigenspace $E^{\lambda}$ at $\overline{\mathbf{x}}$, and $\mathcal{C}$ is the graph of a strictly increasing continuous function of the first coordinate on an interval. Any endpoints of $\mathcal{C}$ in the interior of $\mathcal{R}$ are either fixed points or minimal period-two points. In the latter case, the set of endpoints of $\mathcal{C}$ is a minimal period-two orbit of $T$. 
Theorem 5 (Kulenović \& Merino) Let $\mathcal{I}_{1}, \mathcal{I}_{2}$ be intervals in $\mathbb{R}$ with endpoints $a_{1}, a_{2}$ and $b_{1}, b_{2}$ with endpoints respectively, with $a_{1}<a_{2}$ and $b_{1}<b_{2}$, where $-\infty \leq a_{1}<a_{2} \leq \infty$ and $-\infty \leq b_{1}<b_{2} \leq \infty$. Let $T$ be a competitive map on a rectangle $\mathcal{R}=\mathcal{I}_{1} \times \mathcal{I}_{2}$ and $\overline{\mathbf{x}} \in \operatorname{int}(\mathcal{R})$. Suppose that the following hypotheses are satisfied:

1. $T(\operatorname{int}(\mathcal{R})) \subset \operatorname{int}(\mathcal{R})$ and $T$ is strongly competitive on $\operatorname{int}(\mathcal{R})$.

2. The point $\overline{\mathbf{x}}$ is the only fixed point of $T$ in $\left(Q_{1}(\overline{\mathbf{x}}) \cup Q_{3}(\overline{\mathbf{x}})\right) \cap \operatorname{int}(\mathcal{R})$.

3. The map $T$ is continuously differentiable in a neighborhood of $\overline{\mathbf{x}}$.

4. At least one of the following statements is true:

a. $T$ has no minimal period two orbits in $\left(Q_{1}(\overline{\mathbf{x}}) \cup Q_{3}(\overline{\mathbf{x}})\right) \cap \operatorname{int}(\mathcal{R})$.

b. $\operatorname{det} J_{T}(\overline{\mathbf{x}})>0$ and $T(\mathbf{x})=\overline{\mathbf{x}}$ only for $\mathbf{x}=\overline{\mathbf{x}}$

5. $\overline{\mathbf{x}}$ is a saddle point.

Then the following statements are true.

(i) The stable manifold $\mathcal{W}^{s}(\overline{\mathbf{x}})$ is connected and it is the graph of a continuous increasing curve with endpoints in $\partial \mathcal{R}$. $\operatorname{int}(\mathcal{R})$ is divided by the closure of $\mathcal{W}^{s}(\overline{\mathbf{x}})$ into two invariant connected regions $\mathcal{W}_{+}$("below the stable set"), and $\mathcal{W}_{-}$("above the stable set"), where

$$
\begin{aligned}
& \mathcal{W}_{-}:=\left\{\mathbf{x} \in \mathcal{R} \backslash \mathcal{W}^{s}(\overline{\mathbf{x}}): \exists \mathbf{x}^{\prime} \in \mathcal{W}^{s}(\overline{\mathbf{x}}) \text { with } \mathbf{x} \preceq_{s e} \mathbf{x}^{\prime}\right\} \\
& \mathcal{W}_{+}:=\left\{\mathbf{x} \in \mathcal{R} \backslash \mathcal{W}^{s}(\overline{\mathbf{x}}): \exists \mathbf{x}^{\prime} \in \mathcal{W}^{s}(\overline{\mathbf{x}}) \text { with } \mathbf{x}^{\prime} \preceq_{s e} \mathbf{x}\right\}
\end{aligned}
$$

(ii) The unstable manifold $\mathcal{W}^{u}(\overline{\mathbf{x}})$ is connected, and it is the graph of a continuous decreasing curve.

(iii) For every $\mathbf{x} \in \mathcal{W}_{+}, T^{n}(\mathbf{x})$ eventually enters the interior of the invariant set $Q_{4}(\overline{\mathbf{x}}) \cap \mathcal{R}$, and for every $\mathbf{x} \in \mathcal{W}_{-}, T^{n}(\mathbf{x})$ eventually enters the interior of the invariant set $Q_{2}(\overline{\mathbf{x}}) \cap \mathcal{R}$.

(iv) Let $\mathbf{m} \in Q_{2}(\overline{\mathbf{x}})$ and $\mathbf{M} \in Q_{4}(\overline{\mathbf{x}})$ be the endpoints of $\mathcal{W}^{u}(\overline{\mathbf{x}})$, where $\mathbf{m} \preceq_{s e} \overline{\mathbf{x}} \preceq_{s e} \mathbf{M}$. For every $\mathbf{x} \in \mathcal{W}$ - and every $\mathbf{z} \in \mathcal{R}$ such that $\mathbf{m} \preceq_{\text {se }} z$, there exists $m \in \mathbb{N}$ such that $T^{m}(\mathbf{x}) \preceq_{\text {se }} z$, and for every $\mathbf{x} \in \mathcal{W}_{+}$and every $\mathbf{z} \in \mathcal{R}$ such that $\mathbf{z} \preceq_{\text {se }} \mathbf{M}$, there exists $m \in \mathbb{N}$ such that $\mathbf{M} \preceq_{s e} T^{m}(\mathbf{x})$.

\section{Linearized stability analysis}

\section{Lemma 1}

(i) If $\beta_{2} \gamma_{1}-A_{1} A_{2} \leq 0$, then System (2) has a unique equilibrium point $E_{0}=(0,0)$.

(ii) If $\beta_{2} \gamma_{1}-A_{1} A_{2}>0$, then System (2) has two equilibrium points $E_{0}$ and $E_{+}=(\bar{x}, \bar{y})$, $\bar{x}>0, \bar{y}>0$.

Proof The equilibrium point $E(\bar{x}, \bar{y})$ of System (2) satisfies the following system of equations:

$$
\bar{x}=\frac{\gamma_{1} \bar{y}}{A_{1}+\bar{x}}, \quad \bar{y}=\frac{\beta_{2} \bar{x}}{A_{2}+B_{2} \bar{x}+\bar{y}} .
$$

It is easy to see that $E_{0}=(0,0)$ is one equilibrium point for all values of the parameters, and $E_{+}=(\bar{x}, \bar{y})$ is a positive equilibrium point if $\beta_{2} \gamma_{1}-A_{1} A_{2}>0$. Indeed, substituting $\bar{y}$ from the first equation in (4) in the second equation in (4), we obtain that $\bar{x}$ satisfies the following equation:

$$
f(x)=x^{3}+\left(2 A_{1}+B_{2} \gamma_{1}\right) x^{2}+\left(A_{1}^{2}+A_{1} B_{2} \gamma_{1}+A_{2} \gamma_{1}\right) x+\gamma_{1}\left(A_{1} A_{2}-\beta_{2} \gamma_{1}\right)=0 .
$$


By using Descartes' theorem, we have that equation (5) has one positive equilibrium if the condition

$$
\beta_{2} \gamma_{1}-A_{1} A_{2}>0
$$

is satisfied, i.e., $\beta_{2} \gamma_{1}>A_{1} A_{2}$.

\section{Theorem 6}

(i) If $\beta_{2} \gamma_{1}<A_{1} A_{2}$, then $E_{0}$ is locally asymptotically stable.

(ii) If $\beta_{2} \gamma_{1}=A_{1} A_{2}$, then $E_{0}$ is non-hyperbolic.

(iii) If $\beta_{2} \gamma_{1}>A_{1} A_{2}$, then $E_{0}$ is a repeller.

Proof The map $T$ associated to System (2) is of the form (3). The Jacobian matrix of $T$ at the equilibrium $E=(\bar{x}, \bar{y})$ is

$$
J_{T}(\bar{x}, \bar{y})=\left(\begin{array}{cc}
-\frac{\gamma_{1} \bar{y}}{\left(A_{1}+\bar{x}\right)^{2}} & \frac{\gamma_{1}}{A_{1}+\bar{x}} \\
\frac{\beta_{2}\left(A_{2}+\bar{y}\right)}{\left(A_{2}+B_{2} \bar{x}+\bar{y}\right)^{2}} & -\frac{\beta_{2} \bar{x}}{\left(A_{2}+B_{2} \bar{x}+\bar{y}\right)^{2}}
\end{array}\right)
$$

and

$$
J_{T}(0,0)=\left(\begin{array}{cc}
0 & \frac{\gamma_{1}}{A_{1}} \\
\frac{\beta_{2}}{A_{2}} & 0
\end{array}\right) .
$$

The corresponding characteristic equation has the following form:

$$
\lambda^{2}-\frac{\beta_{2} \gamma_{1}}{A_{1} A_{2}}=0,
$$

from which $\lambda_{1,2}= \pm \sqrt{\frac{\beta_{2} \gamma_{1}}{A_{1} A_{2}}}$.

(i) If $\beta_{2} \gamma_{1}<A_{1} A_{2}$, then $\left|\lambda_{1,2}\right|<1$, i.e., $E_{0}$ is locally asymptotically stable.

(ii) If $\beta_{2} \gamma_{1}=A_{1} A_{2}$, then $\left|\lambda_{1,2}\right|=1$, which implies that $E_{0}$ is non-hyperbolic.

(iii) If $\beta_{2} \gamma_{1}>A_{1} A_{2}$, then $\left|\lambda_{1,2}\right|>1$, which implies that $E_{0}$ is a repeller.

\section{Theorem 7}

(1) Assume that $\beta_{2} \gamma_{1}>A_{1} A_{2}$ and

$$
\beta_{2} \gamma_{1}-A_{1} A_{2}>-B_{2}\left[A_{1}^{2}+\gamma_{1}\left(A_{2}-A_{1} B_{2}\right)\right] .
$$

Then the positive equilibrium $E_{+}$is a saddle point.

(2) Assume that

$$
0<\beta_{2} \gamma_{1}-A_{1} A_{2}=-B_{2}\left[A_{1}^{2}+\gamma_{1}\left(A_{2}-A_{1} B_{2}\right)\right] .
$$

Then the positive equilibrium $E_{+}$is a non-hyperbolic point and

$$
\bar{x}=-A_{1}+\sqrt{\gamma_{1}\left(A_{1} B_{2}-A_{2}\right)}, \quad \bar{y}=\frac{\left(-A_{1}+\sqrt{\gamma_{1}\left(A_{1} B_{2}-A_{2}\right)}\right) \sqrt{\gamma_{1}\left(A_{1} B_{2}-A_{2}\right)}}{\gamma_{1}} .
$$


(3) Assume that

$$
0<\beta_{2} \gamma_{1}-A_{1} A_{2}<-B_{2}\left[A_{1}^{2}+\gamma_{1}\left(A_{2}-A_{1} B_{2}\right)\right]
$$

Then the positive equilibrium $E_{+}$is locally asymptotically stable.

Proof The Jacobian matrix of $T$ at the equilibrium $E_{+}=(\bar{x}, \bar{y})$ is of the form (7) and the corresponding characteristic equation has the following form:

$$
\lambda^{2}-p \lambda+q=0
$$

where

$$
\begin{aligned}
p & =\operatorname{Tr} J_{T}\left(E_{+}\right)=-\frac{\bar{x}}{A_{1}+\bar{x}}-\frac{\bar{y}}{A_{2}+B_{2} \bar{x}+\bar{y}}=-\frac{\bar{x}^{2}}{\gamma_{1} \bar{y}}-\frac{\bar{y}^{2}}{\beta_{2} \bar{x}}=\frac{-A_{2} \bar{x}-B_{2} \bar{x}^{2}-2 \overline{x y}-A_{1} \bar{y}}{\left(A_{1}+\bar{x}\right)\left(A_{2}+B_{2} \bar{x}+\bar{y}\right)}<0, \\
q & =\operatorname{det} J_{T}\left(E_{+}\right)=\frac{\overline{x y}}{\left(A_{1}+\bar{x}\right)\left(A_{2}+B_{2} \bar{x}+\bar{y}\right)}-\frac{\beta_{2} \gamma_{1}\left(A_{2}+\bar{y}\right)}{\left(A_{1}+\bar{x}\right)\left(A_{2}+B_{2} \bar{x}+\bar{y}\right)^{2}} \\
& =\frac{\overline{x y}}{\left(A_{1}+\bar{x}\right)\left(A_{2}+B_{2} \bar{x}+\bar{y}\right)}-\frac{A_{2}+\bar{y}}{A_{2}+B_{2} \bar{x}+\bar{y}}=\frac{\overline{x y}}{\beta_{2} \gamma_{1}}-\frac{\bar{y}\left(A_{2}+\bar{y}\right)}{\beta_{2} \bar{x}} \\
& =\frac{\bar{y}\left(\bar{x}^{2}-A_{2} \gamma_{1}-\gamma_{1} \bar{y}\right)}{\beta_{2} \gamma_{1} \bar{x}}=\frac{\bar{y}\left(-A_{2} \gamma_{1}-A_{1} \bar{x}\right)}{\beta_{2} \gamma_{1} \bar{x}}<0 .
\end{aligned}
$$

Hence, for $E_{+}=(\bar{x}, \bar{y})$, we have $p<0, q<0$, so $p^{2}-4 q>0$. Since

$$
\begin{aligned}
p-q-1 & =-\frac{\bar{x}^{2}}{\gamma_{1} \bar{y}}-\frac{\bar{y}^{2}}{\beta_{2} \bar{x}}-\frac{\overline{x y}}{\beta_{2} \gamma_{1}}+\frac{\bar{y}\left(A_{2}+\bar{y}\right)}{\beta_{2} \bar{x}}-1 \stackrel{(4)}{=}-\frac{\bar{x}^{2}}{\gamma_{1} \bar{y}}-\frac{\bar{y}^{2}}{\beta_{2} \bar{x}}-\frac{\overline{x y}}{\beta_{2} \gamma_{1}}+\left(1-\frac{B_{2} \bar{y}}{\beta_{2}}\right)-1 \\
& =-\frac{\bar{x}^{2}}{\gamma_{1} \bar{y}}-\frac{\bar{y}^{2}}{\beta_{2} \bar{x}}-\frac{\overline{x y}}{\beta_{2} \gamma_{1}}-\frac{B_{2} \bar{y}}{\beta_{2}}<0,
\end{aligned}
$$

we obtain

$$
|p|\left\{\begin{array}{l}
>|1+q|, \\
=|1+q|, \\
<|1+q|
\end{array} \Leftrightarrow 1+p+q\left\{\begin{array}{l}
<0, \\
=0, \\
>0 .
\end{array}\right.\right.
$$

Similarly,

$$
\begin{aligned}
1+p+q & =1-\frac{\bar{x}}{A_{1}+\bar{x}}-\frac{\bar{y}}{A_{2}+B_{2} \bar{x}+\bar{y}}+\frac{\overline{x y}}{\left(A_{1}+\bar{x}\right)\left(A_{2}+B_{2} \bar{x}+\bar{y}\right)}-\frac{A_{2}+\bar{y}}{A_{2}+B_{2} \bar{x}+\bar{y}} \\
& =-\frac{A_{2} \bar{x}+\bar{y}\left(A_{1}+\bar{x}\right)-A_{1} B_{2} \bar{x}}{\left(\bar{x}+A_{1}\right)\left(A_{2}+B_{2} \bar{x}+\bar{y}\right)} \\
& \stackrel{(4)}{=}-\frac{\bar{x}}{\gamma_{1}\left(\bar{x}+A_{1}\right)\left(A_{2}+B_{2} \bar{x}+\bar{y}\right)} \phi(\bar{x}),
\end{aligned}
$$

where

$$
\phi(x)=x^{2}+2 A_{1} x+A_{1}^{2}+\gamma_{1}\left(A_{2}-A_{1} B_{2}\right), \quad \text { for } x>0 .
$$


Now, for the positive equilibrium, it holds

$$
\begin{aligned}
& 1+p+q>0 \quad \Leftrightarrow \quad \phi(\bar{x})<0, \\
& 1+p+q=0 \quad \Leftrightarrow \quad \phi(\bar{x})=0, \\
& 1+p+q<0 \quad \Leftrightarrow \quad \phi(\bar{x})>0 .
\end{aligned}
$$

If $A_{1}^{2}+\gamma_{1}\left(A_{2}-A_{1} B_{2}\right) \geq 0$, then $\phi(x)>0$ for all $x>0$, which implies that $E_{+}$is a saddle point. If $A_{1}^{2}+\gamma_{1}\left(A_{2}-A_{1} B_{2}\right)<0$, then $\phi(x)=0$ for $x_{ \pm}=-A_{1} \pm \sqrt{\gamma_{1}\left(A_{1} B_{2}-A_{2}\right)}\left(x_{-}<0, x_{+}>0\right)$.

Now we have three cases: $x_{+}<\bar{x}, x_{+}=\bar{x}$ or $\bar{x}<x_{+}$. Functions $f(x)$ and $\phi(x)$ are increasing for $x>0$.

(1) If $x_{+}<\bar{x}$, then $0=\phi\left(x_{+}\right)<\phi(\bar{x})$, i.e., $1+p+q<0$ and $f\left(x_{+}\right)<f(\bar{x})=0$. So,

$$
\begin{aligned}
& f\left(x_{+}\right)=f(\left.-A_{1}+\sqrt{\gamma_{1}\left(A_{1} B_{2}-A_{2}\right)}\right)<0 \\
& \Leftrightarrow \quad\left(-A_{1}+\sqrt{\gamma_{1}\left(A_{1} B_{2}-A_{2}\right)}\right)^{3}+\left(2 A_{1}+B_{2} \gamma_{1}\right)\left(-A_{1}+\sqrt{\gamma_{1}\left(A_{1} B_{2}-A_{2}\right)}\right)^{2} \\
& \quad+\left(A_{1}^{2}+A_{1} B_{2} \gamma_{1}+A_{2} \gamma_{1}\right)\left(-A_{1}+\sqrt{\gamma_{1}\left(A_{1} B_{2}-A_{2}\right)}\right)+\gamma_{1}\left(A_{1} A_{2}-\beta_{2} \gamma_{1}\right)<0
\end{aligned}
$$

from which it follows

$$
\gamma_{1} B_{2}\left(A_{1} B_{2}-A_{2}\right)<\left(\beta_{2} \gamma_{1}-A_{1} A_{2}\right)+A_{1}^{2} B_{2}
$$

i.e.,

$$
\beta_{2} \gamma_{1}>\left(A_{1}-\gamma_{1} B_{2}\right)\left(A_{2}-A_{1} B_{2}\right)
$$

Now we have

$$
\beta_{2} \gamma_{1}-A_{1} A_{2}>-B_{2}\left[A_{1}^{2}+\gamma_{1}\left(A_{2}-A_{1} B_{2}\right)\right]
$$

so we can see that the conditions (8) and (6) are sufficient for $E_{+}=(\bar{x}, \bar{y})$ to be a saddle point.

(2) If $x_{+}=\bar{x}$, then $0=\phi\left(x_{+}\right)=\phi(\bar{x})$, hence $1+p+q=0$, i.e.,

$$
f\left(x_{+}\right)=f(\bar{x})=f\left(-A_{1}+\sqrt{\gamma_{1}\left(A_{1} B_{2}-A_{2}\right)}\right)=0,
$$

from which

$$
\beta_{2} \gamma_{1}=\left(A_{1}-\gamma_{1} B_{2}\right)\left(A_{2}-A_{1} B_{2}\right)
$$

If conditions (12) and (6) are satisfied, then

$$
\beta_{2} \gamma_{2}-A_{1} A_{2}=-B_{2}\left[A_{1}^{2}+\gamma_{1}\left(A_{2}-A_{1} B_{2}\right)\right]>0
$$

holds, i.e., $E_{+}=(\bar{x}, \bar{y})$ is a non-hyperbolic point of the form

$$
\bar{x}=x_{+}=-A_{1}+\sqrt{\gamma_{1}\left(A_{1} B_{2}-A_{2}\right)}, \quad \bar{y}=\frac{\left(-A_{1}+\sqrt{\gamma_{1}\left(A_{1} B_{2}-A_{2}\right)}\right) \sqrt{\gamma_{1}\left(A_{1} B_{2}-A_{2}\right)}}{\gamma_{1}} .
$$


(3) If $\bar{x}<x_{+}$, then $\phi(\bar{x})<\phi\left(x_{+}\right)=0$ and

$$
0=f(\bar{x})<f\left(x_{+}\right)=f\left(-A_{1}+\sqrt{\gamma_{1}\left(A_{1} B_{2}-A_{2}\right)}\right),
$$

from which

$$
\beta_{2} \gamma_{1}<\left(A_{1}-\gamma_{1} B_{2}\right)\left(A_{2}-A_{1} B_{2}\right) .
$$

Hence, if conditions (13) and (6) are satisfied, then

$$
0<\beta_{2} \gamma_{2}-A_{1} A_{2}<-B_{2}\left[A_{1}^{2}+\gamma_{1}\left(A_{2}-A_{1} B_{2}\right)\right]
$$

holds, so $E_{+}$is a locally asymptotically stable.

\section{Periodic character of solutions}

In this section, we give the existence and local stability of period-two solutions.

Lemma 2 Assume that $\beta_{2} \gamma_{1}>A_{1} A_{2}$. Then System (2) has the following minimal period-two solutions:

$$
A_{0}=\left(0, \frac{\beta_{2} \gamma_{1}-A_{1} A_{2}}{\gamma_{1} B_{2}}\right) \text { and } B_{0}=\left(\frac{\beta_{2} \gamma_{1}-A_{1} A_{2}}{A_{1} B_{2}}, 0\right) \text {. }
$$

If

$$
0<\beta_{2} \gamma_{1}-A_{1} A_{2}=-B_{2}\left[A_{1}^{2}+\gamma_{1}\left(A_{2}-A_{1} B_{2}\right)\right] \text {, }
$$

then System (2) has an infinite number of minimal period-two solutions of the form

$$
\begin{aligned}
& A_{x}=\left(x, \frac{\beta_{2} \gamma_{1}-A_{1} A_{2}-x A_{1} B_{2}}{\gamma_{1} B_{2}}\right), \\
& B_{x}=\left(\frac{\beta_{2} \gamma_{1}-A_{1} A_{2}-x A_{1} B_{2}}{B_{2}\left(x+A_{1}\right)}, \frac{-x \beta_{2} \gamma_{1}}{\left(A_{1}-\gamma_{1} B_{2}\right)\left(x+A_{1}\right)}\right)
\end{aligned}
$$

for $x \in\left[0, \frac{\beta_{2} \gamma_{1}-A_{1} A_{2}}{A_{1} B_{2}}\right]$, located along the line

$$
\mathcal{H}=\left\{(x, y): x A_{1}+\gamma_{1} y+A_{1}^{2}+\gamma_{1}\left(A_{2}-A_{1} B_{2}\right)=0, x \in\left[0, \frac{\beta_{2} \gamma_{1}-A_{1} A_{2}}{A_{1} B_{2}}\right]\right\} .
$$

Proof The second iterate of $T$ is (25). Equilibrium curves of the map $T^{2}(x, y)$ are

$$
C_{1 T^{2}}=\left\{(x, y) \in[0, \infty)^{2}: x \beta_{2} \gamma_{1}\left(x+A_{1}\right)=x\left(y+A_{2}+x B_{2}\right)\left(A_{1}^{2}+x A_{1}+y \gamma_{1}\right)\right\}
$$

and

$$
\begin{aligned}
C_{2 T^{2}}= & \left\{(x, y) \in[0, \infty)^{2}: y \beta_{2} \gamma_{1}\left(y+A_{2}+x B_{2}\right)=y\left(A_{1} A_{2}^{2}+x^{2} \beta_{2}+x A_{2}^{2}+x^{2} A_{2} B_{2}+x y A_{2}\right.\right. \\
& \left.\left.+x \beta_{2} A_{1}+y A_{1} A_{2}+y^{2} \gamma_{1} B_{2}+y \gamma_{1} A_{2} B_{2}+x A_{1} A_{2} B_{2}+x y \gamma_{1} B_{2}^{2}\right)\right\} .
\end{aligned}
$$


We get period-two solutions as the intersection point of equilibrium curves (16) and (17) in the first quadrant. If $x \neq 0, y=0$, then System (16), (17) is reduced to the equation

$$
\beta_{2} \gamma_{1}\left(x+A_{1}\right)=A_{1}\left(A_{2}+x B_{2}\right)\left(x+A_{1}\right)
$$

and the positive solution of this equation is

$$
x=\frac{\beta_{2} \gamma_{1}-A_{1} A_{2}}{A_{1} B_{2}}>0, \quad \text { for } \beta_{2} \gamma_{1}-A_{1} A_{2}>0 .
$$

If $x=0, y \neq 0$, then System (16), (17) is reduced to the equation

$$
\beta_{2} \gamma_{1}\left(y+A_{2}\right)=\left(y+A_{2}\right)\left(A_{1} A_{2}+y \gamma_{1} B_{2}\right)
$$

with the positive solution

$$
y=\frac{\beta_{2} \gamma_{1}-A_{1} A_{2}}{\gamma_{1} B_{2}}>0, \quad \text { for } \beta_{2} \gamma_{1}-A_{1} A_{2}>0 .
$$

On the other hand, if $x>0, y>0$, then we have

$$
\left.\begin{array}{rl}
\beta_{2} \gamma_{1}\left(x+A_{1}\right)=(y+ & \left.A_{2}+x B_{2}\right)\left(A_{1}^{2}+x A_{1}+y \gamma_{1}\right) \\
\beta_{2} \gamma_{1}\left(y+A_{2}+x B_{2}\right)= & A_{1} A_{2}^{2}+x^{2} \beta_{2}+x A_{2}^{2}+x^{2} A_{2} B_{2}+x y A_{2}+x \beta_{2} A_{1}+y A_{1} A_{2} \\
& +y^{2} \gamma_{1} B_{2}+y \gamma_{1} A_{2} B_{2}+x A_{1} A_{2} B_{2}+x y \gamma_{1} B_{2}^{2}
\end{array}\right\},
$$

that is

$$
\left(x+A_{1}\right)\left(\beta_{2} \gamma_{1}-A_{1} A_{2}\right)=\left(y+x B_{2}\right)\left(A_{1}^{2}+x A_{1}+y \gamma_{1}\right)+y \gamma_{1} A_{2}
$$

and

$$
\begin{aligned}
x^{2} & \beta_{2}+x A_{2}^{2}+x^{2} A_{2} B_{2}+x y A_{2}+x \beta_{2} A_{1}+y^{2} \gamma_{1} B_{2}+y \gamma_{1} A_{2} B_{2}+x y \gamma_{1} B_{2}^{2} \\
& =\left(y+x B_{2}+A_{2}\right)\left(\beta_{2} \gamma_{1}-A_{1} A_{2}\right) .
\end{aligned}
$$

Therefore, it must be $\left(\beta_{2} \gamma_{1}-A_{1} A_{2}\right)>0$ in order to get any positive solution. By eliminating the term $\left(\beta_{2} \gamma_{1}-A_{1} A_{2}\right)$ from (18) and using condition (9), we get

$$
\left(y+x B_{2}+A_{1} B_{2}\right)\left(y \gamma_{1}+x A_{1}+A_{1}^{2}+\gamma_{1} A_{2}-\gamma_{1} A_{1} B_{2}\right)=0,
$$

which implies

$$
y \gamma_{1}+x A_{1}+A_{1}^{2}+\gamma_{1}\left(A_{2}-A_{1} B_{2}\right)=0
$$

hence

$$
y=-\frac{1}{\gamma_{1}}\left(x A_{1}+A_{1}^{2}+\gamma_{1}\left(A_{2}-A_{1} B_{2}\right)\right), \quad \gamma_{1} \neq 0 .
$$


Now, by eliminating $y$ and the term $\left(A_{1} A_{2}-\beta_{2} \gamma_{1}\right)$ from (19), we get the identity

$$
\left(x+A_{1}\right)\left(x+A_{1}-\gamma_{1} B_{2}\right) \frac{\beta_{2} \gamma_{1}-\left(A_{2}-A_{1} B_{2}\right)\left(A_{1}-\gamma_{1} B_{2}\right)}{\gamma_{1}}=0 .
$$

If $x=\gamma_{1} B_{2}-A_{1}$, we have

$$
y=-\frac{1}{\gamma_{1}}\left(x A_{1}+A_{1}^{2}+\gamma_{1}\left(A_{2}-A_{1} B_{2}\right)\right)=-A_{2}<0, \quad \gamma_{1} \neq 0 .
$$

So, periodic solutions are located along line (15) with endpoints given by (14) using conditions (9). It is easy to see that $A_{x}, B_{x} \in \mathcal{H}$ if $\beta_{2} \gamma_{1}-A_{1} A_{2}=-B_{2}\left[A_{1}^{2}+\gamma_{1}\left(A_{2}-A_{1} B_{2}\right)\right]$.

Let $(x, y) \in \mathcal{H}$, then the corresponding Jacobian matrix of the map $T^{2}$ has the following form:

$$
J_{T^{2}}^{\mathcal{H}}(x, y)=\left(\begin{array}{ll}
a & b \\
c & d
\end{array}\right)
$$

where $a:=F_{x}(x, y), b:=F_{y}(x, y), c:=G_{x}(x, y), d:=G_{y}(x, y)$.

Lemma 3 Assume that $0<\beta_{2} \gamma_{1}-A_{1} A_{2}=-B_{2}\left[A_{1}^{2}+\gamma_{1}\left(A_{2}-A_{1} B_{2}\right)\right]$. Then the following statements are true.

(a) The points $A_{x}, B_{x} \in \mathcal{H}$ are non-hyperbolic fixed points for the map $T^{2}$, and both of them have eigenvalues $\lambda_{1}=1$ and $\lambda_{2} \in(0,1)$.

(b) Eigenvectors corresponding to the eigenvalues $\lambda_{1}$ and $\lambda_{2}$ are not parallel to coordinate axes.

Proof

(a) From (15) we have $y_{\mathcal{H}}^{\prime}(x)=-\frac{A_{1}}{\gamma_{1}}<0$. Since

$$
\mathcal{H}=\left\{(x, y) \in[0, \infty)^{2}: F(x, y)=x\right\}=\left\{(x, y) \in[0, \infty)^{2}: G(x, y)=y\right\},
$$

by implicit differentiation of equations $F(x, y)=x$ and $G(x, y)=y$ at the point $(x, y) \in \mathcal{H}$, we obtain

$$
y_{\mathcal{H}}^{\prime}(x)=\frac{1-a}{b}=\frac{c}{1-d}=-\frac{A_{1}}{\gamma_{1}}<0 .
$$

Since $a>0, b<0, c<0$ and $d>0$, from (22), we get

$$
0<a<1 \text { and } 0<d<1 \text {. }
$$

The characteristic polynomial of the matrix (21) at the point $(x, y) \in \mathcal{H}$ is of the form

$$
P(\lambda)=\lambda^{2}-(a+d) \lambda+(a d-b c) .
$$

Now, using (22) we have $(1-a)(1-d)=b c$, and since

$$
P(1)=1-(a+d)+(a d-b c)=0,
$$


we get $\lambda_{1}=1$, and due to Vieta's formulas and condition (23), it follows

$$
0<\lambda_{1}+\lambda_{2}=1+\lambda_{2}=a+d<2,
$$

i.e., $0<\lambda_{2}<1$.

(b) Eigenvectors corresponding to the eigenvalues $\lambda_{1}$ and $\lambda_{2}$ are $\mathbf{v}_{1}=(1-d, c)$ and $\mathbf{v}_{2}=(a-1, c)$. By condition (23) it is easy to see that these vectors are not parallel to the coordinate axes.

Lemma 4 The periodic points $A_{0}$ and $B_{0}$ given by (14) are

(a) locally asymptotically stable if $\beta_{2} \gamma_{1}-A_{1} A_{2}>-B_{2}\left[A_{1}^{2}+\gamma_{1}\left(A_{2}-A_{1} B_{2}\right)\right]$ and $\beta_{2} \gamma_{1}>A_{1} A_{2}$,

(b) non-hyperbolic if $0<\beta_{2} \gamma_{1}-A_{1} A_{2}=-B_{2}\left[A_{1}^{2}+\gamma_{1}\left(A_{2}-A_{1} B_{2}\right)\right]$,

(c) saddle points if $0<\beta_{2} \gamma_{1}-A_{1} A_{2}<-B_{2}\left[A_{1}^{2}+\gamma_{1}\left(A_{2}-A_{1} B_{2}\right)\right]$.

Proof We have that

$$
J_{T^{2}}\left(\frac{\beta_{2} \gamma_{1}-A_{1} A_{2}}{A_{1} B_{2}}, 0\right)=\left(\begin{array}{cc}
\frac{A_{1} A_{2}}{\beta_{2} \gamma_{1}} & \frac{\left(\beta_{2} \gamma_{1}-A_{1} A_{2}\right)\left(A_{1}^{2} A_{2}-A_{1}^{3} B_{2}-\beta_{2} \gamma_{1}^{2} B_{2}-\beta_{2} \gamma_{1} A_{1}\right)}{\beta_{2} \gamma_{1} A_{1} B_{2}\left(A_{1}^{2} B_{2}+\beta_{2} \gamma_{1}-A_{1} A_{2}\right)} \\
0 & \frac{\beta_{2} \gamma_{1}^{2} A_{1} B_{2}^{2}}{\left(A_{1}^{2} B_{2}+\beta_{2} \gamma_{1}-A_{1} A_{2}\right)\left(\beta_{2} \gamma_{1}-A_{1} A_{2}+\gamma_{1} A_{2} B_{2}\right)}
\end{array}\right)
$$

and characteristic eigenvalues are

$$
\lambda_{1}=\frac{A_{1} A_{2}}{\beta_{2} \gamma_{1}}<1 \quad \text { and } \quad \lambda_{2}=\frac{\beta_{2} \gamma_{1}^{2} A_{1} B_{2}^{2}}{\left(\beta_{2} \gamma_{1}-A_{1} A_{2}+\gamma_{1} A_{2} B_{2}\right)\left(B_{2} A_{1}^{2}-A_{2} A_{1}+\beta_{2} \gamma_{1}\right)} .
$$

Now,

$$
\begin{aligned}
\left|\lambda_{2}\right|<1 & \\
& \Leftrightarrow \quad \beta_{2} \gamma_{1}^{2} A_{1} B_{2}^{2}<\left(\beta_{2} \gamma_{1}-A_{1} A_{2}+\gamma_{1} A_{2} B_{2}\right)\left(B_{2} A_{1}^{2}-A_{2} A_{1}+\beta_{2} \gamma_{1}\right) \\
& \Leftrightarrow \quad\left(\beta_{2} \gamma_{1}-A_{1} A_{2}+\gamma_{1} A_{2} B_{2}\right)\left(B_{2} A_{1}^{2}-A_{2} A_{1}+\beta_{2} \gamma_{1}\right)-\beta_{2} \gamma_{1}^{2} A_{1} B_{2}^{2}>0 \\
& \Leftrightarrow \quad\left(\beta_{2} \gamma_{1}-A_{1} A_{2}\right)\left(A_{1}^{2} B_{2}+\beta_{2} \gamma_{1}-A_{1} A_{2}-\gamma_{1} A_{1} B_{2}^{2}+\gamma_{1} A_{2} B_{2}\right)>0 \\
& \Leftrightarrow \quad\left(A_{1}^{2} B_{2}+\beta_{2} \gamma_{1}-A_{1} A_{2}-\gamma_{1} A_{1} B_{2}^{2}+\gamma_{1} A_{2} B_{2}\right)>0 \\
& \Leftrightarrow \quad \beta_{2} \gamma_{1}-A_{1} A_{2}>-B_{2}\left[A_{1}^{2}+\gamma_{1}\left(A_{2}-A_{1} B_{2}\right)\right] .
\end{aligned}
$$

Therefore,

$$
\begin{aligned}
& \left|\lambda_{2}\right|<1 \quad \Leftrightarrow \quad \beta_{2} \gamma_{1}-A_{1} A_{2}>-B_{2}\left[A_{1}^{2}+\gamma_{1}\left(A_{2}-A_{1} B_{2}\right)\right], \\
& \left|\lambda_{2}\right|=1 \quad \Leftrightarrow \quad 0<\beta_{2} \gamma_{1}-A_{1} A_{2}=-B_{2}\left[A_{1}^{2}+\gamma_{1}\left(A_{2}-A_{1} B_{2}\right)\right], \\
& \left|\lambda_{2}\right|>1 \quad \Leftrightarrow \quad \beta_{2} \gamma_{1}-A_{1} A_{2}<-B_{2}\left[A_{1}^{2}+\gamma_{1}\left(A_{2}-A_{1} B_{2}\right)\right] .
\end{aligned}
$$

On the other hand, we have

$$
J_{T^{2}}\left(0, \frac{\beta_{2} \gamma_{1}-A_{1} A_{2}}{\gamma_{1} B_{2}}\right)=\left(\begin{array}{cc}
\frac{\beta_{2} \gamma_{1}^{2} A_{1} B_{2}^{2}}{\left(A_{1}^{2} B_{2}+\beta_{2} \gamma_{1}-A_{1} A_{2}\right)\left(\beta_{2} \gamma_{1}-A_{1} A_{2}+\gamma_{1} A_{2} B_{2}\right)} & 0 \\
\frac{\left(\beta_{2} \gamma_{1}-A_{1} A_{2}\right)\left(A_{1} A_{2}^{2}-\gamma_{1} A_{2}^{2} B_{2}-\beta_{2} \gamma_{1} A_{2}-\beta_{2} \gamma_{1} A_{1} B_{2}\right)}{\beta_{2} \gamma_{1}^{2} B_{2}\left(\beta_{2} \gamma_{1}-A_{1} A_{2}+\gamma_{1} A_{2} B_{2}\right)} & \frac{A_{1} A_{2}}{\beta_{2} \gamma_{1}}
\end{array}\right)
$$


and the corresponding eigenvalues are

$$
\lambda_{1}=\frac{A_{1} A_{2}}{\beta_{2} \gamma_{1}}<1 \quad \text { and } \quad \lambda_{2}=\frac{\beta_{2} \gamma_{1}^{2} A_{1} B_{2}^{2}}{\left(\beta_{2} \gamma_{1}-A_{1} A_{2}+\gamma_{1} A_{2} B_{2}\right)\left(B_{2} A_{1}^{2}-A_{2} A_{1}+\beta_{2} \gamma_{1}\right)},
$$

so it comes to the same conclusion!

\section{Global results}

In this section, we present the results on the global dynamics of System (2).

Lemma 5 Every solution of System (2) satisfies

1. $x_{n} \leq \frac{\gamma_{1}}{A_{1}} \cdot \frac{\beta_{2}}{B_{2}}, y_{n} \leq \frac{\beta_{2}}{B_{2}}, n=2,3, \ldots$.

2. If $\beta_{2} \gamma_{1}<A_{1} A_{2}$, then $\lim _{n \rightarrow \infty} x_{n}=0, \lim _{n \rightarrow \infty} y_{n}=0$.

The map $T$ satisfies:

3. $T(\mathcal{B}) \subseteq \mathcal{B}$, where $\mathcal{B}=\left[0, \frac{\gamma_{1}}{A_{1}} \cdot \frac{\beta_{2}}{B_{2}}\right] \times\left[0, \frac{\beta_{2}}{B_{2}}\right]$, that is, $\mathcal{B}$ is an invariant box.

4. $T(\mathcal{B})$ is an attracting box, that is $T\left([0, \infty)^{2}\right) \subseteq \mathcal{B}$.

Proof From System (2), we have

$$
\begin{aligned}
& y_{n+1}=\frac{\beta_{2} x_{n}}{A_{2}+B_{2} x_{n}+y_{n}} \leq \frac{\beta_{2} x_{n}}{B_{2} x_{n}}=\frac{\beta_{2}}{B_{2}}, \\
& y_{n+1}=\frac{\beta_{2} x_{n}}{A_{2}+B_{2} x_{n}+y_{n}} \leq \frac{\beta_{2}}{A_{2}} x_{n}, \\
& x_{n+1}=\frac{\gamma_{1} y_{n}}{A_{1}+x_{n}} \leq \frac{\gamma_{1}}{A_{1}} y_{n},
\end{aligned}
$$

for $n=0,1,2, \ldots$, and

$$
x_{n+1} \leq \frac{\gamma_{1}}{A_{1}} y_{n} \leq \frac{\gamma_{1}}{A_{1}} \cdot \frac{\beta_{2}}{B_{2}}
$$

for $n=1,2, \ldots$. Furthermore, we get

$$
x_{n} \leq \frac{\gamma_{1}}{A_{1}} y_{n-1} \leq \frac{\gamma_{1} \beta_{2}}{A_{1} A_{2}} x_{n-2},
$$

i.e.,

$$
x_{2 n} \leq\left(\frac{\gamma_{1} \beta_{2}}{A_{1} A_{2}}\right)^{n} x_{0}, \quad x_{2 n+1} \leq\left(\frac{\gamma_{1} \beta_{2}}{A_{1} A_{2}}\right)^{n} x_{1},
$$

so it follows that $\lim _{n \rightarrow \infty} x_{n}=0, \lim _{n \rightarrow \infty} y_{n}=0$ if $\beta_{2} \gamma_{1}<A_{1} A_{2}$.

Proof of 3 . and 4 . is an immediate checking.

Lemma 6 The map $T^{2}$ is injective and $\operatorname{det} J_{T^{2}}(x, y)>0$, for all $x \geq 0$ and $y \geq 0$.

Proof

(i) Here we prove that map $T$ is injective, which implies that $T^{2}$ is injective. Indeed, $T\left(\begin{array}{l}x_{1} \\ y_{1}\end{array}\right)=T\left(\begin{array}{l}x_{2} \\ y_{2}\end{array}\right)$ implies that

$$
A_{1}\left(y_{1}-y_{2}\right)=x_{1} y_{2}-x_{2} y_{1}, \quad A_{2}\left(x_{1}-x_{2}\right)=x_{2} y_{1}-x_{1} y_{2} .
$$


By solving System (24) with respect to $x_{1}, x_{2}$ or $y_{1}, y_{2}$, we obtain that $\left(x_{1}, y_{1}\right)=\left(x_{2}, y_{2}\right)$.

(ii) The map $T^{2}(x, y)=\left(\begin{array}{l}F(x, y) \\ G(x, y)\end{array}\right)$ is of the form

$$
\begin{aligned}
T^{2}(x, y) & \\
\quad= & \left(\begin{array}{c}
\frac{x \beta_{2} \gamma_{1}\left(x+A_{1}\right)}{\left(y+A_{2}+x B_{2}\right)\left(A_{1}^{2}+x A_{1}+y \gamma_{1}\right)} \\
\frac{y \beta_{2} \gamma_{1}\left(y+A_{2}+x B_{2}\right)}{A_{1} A_{2}^{2}+x^{2} \beta_{2}+x A_{2}^{2}+x^{2} A_{2} B_{2}+x y A_{2}+x \beta_{2} A_{1}+y A_{1} A_{2}+y^{2} \gamma_{1} B_{2}+y \gamma_{1} A_{2} B_{2}+x A_{1} A_{2} B_{2}+x y \gamma_{1} B_{2}^{2}}
\end{array}\right)
\end{aligned}
$$

and

$$
J_{T^{2}}(x, y)=\left(\begin{array}{ll}
F_{x} & F_{y} \\
G_{x} & G_{y}
\end{array}\right),
$$

where

$$
\begin{aligned}
F_{x}= & \beta_{2} \gamma_{1}\left(A_{1}^{3} A_{2}+y A_{1}^{3}+2 x A_{1}^{2} A_{2}+x^{2} A_{1} A_{2}+2 x y^{2} \gamma_{1}+2 x y A_{1}^{2}+x^{2} y A_{1}\right. \\
& \left.+y^{2} \gamma_{1} A_{1}+2 x y \gamma_{1} A_{2}+y \gamma_{1} A_{1} A_{2}+x^{2} y \gamma_{1} B_{2}\right) \\
& /\left(\left(y \gamma_{1}+x A_{1}+A_{1}^{2}\right)^{2}\left(y+A_{2}+x B_{2}\right)^{2}\right), \\
F_{y}= & -\frac{x \beta_{2} \gamma_{1}\left(x+A_{1}\right)\left(2 y \gamma_{1}+x A_{1}+A_{1}^{2}+\gamma_{1} A_{2}+x \gamma_{1} B_{2}\right)}{\left(y \gamma_{1}+x A_{1}+A_{1}^{2}\right)^{2}\left(y+A_{2}+x B_{2}\right)^{2}}, \\
G_{x}= & -y \beta_{2} \gamma_{1}\left(A_{2}^{3}+2 y A_{2}^{2}+y^{2} A_{2}+2 x A_{2}^{2} B_{2}+2 x y \beta_{2}+2 x \beta_{2} A_{2}+y \beta_{2} A_{1}\right. \\
& \left.+x^{2} A_{2} B_{2}^{2}+\beta_{2} A_{1} A_{2}+x^{2} \beta_{2} B_{2}+2 x y A_{2} B_{2}\right) \\
& /\left(A_{1} A_{2}^{2}+x^{2} \beta_{2}+x A_{2}^{2}+x^{2} A_{2} B_{2}+x y A_{2}+x \beta_{2} A_{1}+y A_{1} A_{2}\right. \\
& \left.+y^{2} \gamma_{1} B_{2}+y \gamma_{1} A_{2} B_{2}+x A_{1} A_{2} B_{2}+x y \gamma_{1} B_{2}^{2}\right)^{2}, \\
G_{y}= & \beta_{2} \gamma_{1}\left(x+A_{1}\right)\left(A_{2}^{3}+2 y A_{2}^{2}+y^{2} A_{2}+2 x A_{2}^{2} B_{2}+2 x y \beta_{2}+x \beta_{2} A_{2}+x^{2} A_{2} B_{2}^{2}\right. \\
& \left.+x^{2} \beta_{2} B_{2}+2 x y A_{2} B_{2}\right) \\
& /\left(A_{1} A_{2}^{2}+x^{2} \beta_{2}+x A_{2}^{2}+x^{2} A_{2} B_{2}+x y A_{2}+x \beta_{2} A_{1}+y A_{1} A_{2}\right. \\
& \left.+y^{2} \gamma_{1} B_{2}+y \gamma_{1} A_{2} B_{2}+x A_{1} A_{2} B_{2}+x y \gamma_{1} B_{2}^{2}\right)^{2} .
\end{aligned}
$$

Now, we obtain

$$
\operatorname{det} J_{T^{2}}(x, y)=F_{x} G_{y}-F_{y} G_{x}=U V,
$$

where

$$
\begin{aligned}
U= & \frac{\beta_{2}^{2} \gamma_{1}^{2}\left(x+A_{1}\right)\left(x A_{2}+y A_{1}+A_{1} A_{2}\right)}{\left(y \gamma_{1}+x A_{1}+A_{1}^{2}\right)^{2}\left(y+A_{2}+x B_{2}\right)}>0, \\
V= & \left(A_{1}^{2} A_{2}^{2}+x A_{1} A_{2}^{2}+y A_{1}^{2} A_{2}+x \beta_{2} A_{1}^{2}\right. \\
& \left.+x^{2} \beta_{2} A_{1}+y \gamma_{1} A_{2}^{2}+y^{2} \gamma_{1} A_{2}+x A_{1}^{2} A_{2} B_{2}+x^{2} A_{1} A_{2} B_{2}+x y A_{1} A_{2}+x y \gamma_{1} A_{2} B_{2}\right) \\
& /\left(A_{1} A_{2}^{2}+x^{2} \beta_{2}+x A_{2}^{2}+x^{2} A_{2} B_{2}+x y A_{2}+x \beta_{2} A_{1}+y A_{1} A_{2}\right. \\
& \left.+y^{2} \gamma_{1} B_{2}+y \gamma_{1} A_{2} B_{2}+x A_{1} A_{2} B_{2}+x y \gamma_{1} B_{2}^{2}\right)^{2}>0
\end{aligned}
$$

and the Jacobian matrix of $T^{2}(x, y)$ is invertible for all $x \geq 0$ and $y \geq 0$. 
Corollary 1 The competitive map $T^{2}$ satisfies the condition $(O+)$. Consequently, the sequences $\left\{x_{2 n}\right\},\left\{x_{2 n+1}\right\},\left\{y_{2 n}\right\},\left\{y_{2 n+1}\right\}$ of every solution of System (2) are eventually monotone.

Proof It immediately follows from Lemma 6, Theorem 2 and 3.

Lemma 7 Assume $\beta_{2} \gamma_{1}-A_{1} A_{2}>0$. System (2) has period-two solutions (14) and

(a) If $\left(x_{0}, y_{0}\right)=(x, 0), x>0$, then

$$
\lim _{n \rightarrow \infty} T^{2 n}(x, 0)=\left(\frac{\beta_{2} \gamma_{1}-A_{1} A_{2}}{A_{1} B_{2}}, 0\right)=B_{0}
$$

and

$$
\lim _{n \rightarrow \infty} T^{2 n+1}(x, 0)=\left(0, \frac{\beta_{2} \gamma_{1}-A_{1} A_{2}}{\gamma_{1} B_{2}}\right)=A_{0} .
$$

(b) If $\left(x_{0}, y_{0}\right)=(0, y), y>0$, then

$$
\lim _{n \rightarrow \infty} T^{2 n}(0, y)=\left(0, \frac{\beta_{2} \gamma_{1}-A_{1} A_{2}}{\gamma_{1} B_{2}}\right)=A_{0}
$$

and

$$
\lim _{n \rightarrow \infty} T^{2 n+1}(x, 0)=\left(\frac{\beta_{2} \gamma_{1}-A_{1} A_{2}}{A_{1} B_{2}}, 0\right)=B_{0}
$$

Proof (a) For all $x>0, x \neq \frac{\beta_{2} \gamma_{1}-A_{1} A_{2}}{A_{1} B_{2}}$, we have

$$
\begin{aligned}
& T(x, 0)=\left(0, \frac{\beta_{2} x}{A_{2}+B_{2} x}\right), \quad T^{2}(x, 0)=\left(\frac{\gamma_{1} \beta_{2} x}{A_{1} A_{2}+A_{1} B_{2} x}, 0\right), \\
& T^{3}(x, 0)=\left(0, \frac{\beta_{2}\left(\gamma_{1} \beta_{2}\right) x}{A_{2}\left[A_{1} A_{2}+A_{1} B_{2}\right] x+B_{2} \gamma_{1} \beta_{2} x}\right), \\
& T^{4}(x, 0)=\left(\frac{\left(\gamma_{1} \beta_{2}\right)^{2} x}{\left(A_{1} A_{2}\right)^{2}+A_{1} B_{2} x\left[\left(A_{1} A_{2}\right)+\gamma_{1} \beta_{2}\right]}, 0\right), \\
& T^{5}(x, 0)=\left(0, \frac{\beta_{2}\left(\gamma_{1} \beta_{2}\right)^{2} x}{A_{2}\left[\left(A_{1} A_{2}\right)^{2}+A_{1} B_{2}\left(A_{1} A_{2}\right) x+A_{1} B_{2}\left(\gamma_{1} \beta_{2}\right) x\right]+B_{2}\left(\gamma_{1} \beta_{2}\right)^{2} x}\right), \\
& T^{6}(x, 0)=\left(\frac{\left(\gamma_{1} \beta_{2}\right)^{3} x}{\left(A_{1} A_{2}\right)^{3}+A_{1} B_{2} x\left[\left(A_{1} A_{2}\right)^{2}+A_{1} A_{2} \gamma_{1} \beta_{2}+\left(\beta_{2} \gamma_{1}\right)^{2}\right]}, 0\right)
\end{aligned}
$$

and by induction,

$$
\begin{aligned}
& T^{2 n}(x, 0) \\
& \quad=\left(\frac{\left(\gamma_{1} \beta_{2}\right)^{n} x}{\left(A_{1} A_{2}\right)^{n}+A_{1} B_{2} x\left[\left(A_{1} A_{2}\right)^{n-1}+\left(A_{1} A_{2}\right)^{n-2}\left(\gamma_{1} \beta_{2}\right)^{1}+\cdots+\left(\beta_{2} \gamma_{1}\right)^{n-1}\right]}, 0\right), \\
& T^{2 n+1}(x, 0) \\
& \quad=\left(0, \frac{\beta_{2}\left(\gamma_{1} \beta_{2}\right)^{n} x}{A_{2}\left[\left(A_{1} A_{2}\right)^{n}+A_{1} B_{2}\left(A_{1} A_{2}\right)^{n-1} x+\cdots+A_{1} B_{2}\left(\gamma_{1} \beta_{2}\right)^{n-1} x\right]+B_{2}\left(\gamma_{1} \beta_{2}\right)^{n} x}\right) .
\end{aligned}
$$


Now, we have

$$
\begin{aligned}
\lim _{n \rightarrow \infty} T^{2 n}(x, 0) \\
=\lim _{n \rightarrow \infty}\left(\frac{\left(\gamma_{1} \beta_{2}\right)^{n} x}{\left(A_{1} A_{2}\right)^{n}+A_{1} B_{2} x\left[\left(A_{1} A_{2}\right)^{n-1}+\left(A_{1} A_{2}\right)^{n-2}\left(\gamma_{1} \beta_{2}\right)^{1}+\cdots+\left(\beta_{2} \gamma_{1}\right)^{n-1}\right]}, 0\right) \\
=\lim _{n \rightarrow \infty}\left(\frac{x}{\left(\frac{A_{1} A_{2}}{\beta_{2} \gamma_{1}}\right)^{n}+x\left(\frac{A_{1} B_{2}}{\beta_{2} \gamma_{1}}\right) \frac{1-\left(\frac{A_{1} A_{2}}{\beta_{2} \gamma_{1}}\right)^{n}}{1-\frac{A_{1} A_{2}}{\beta_{2} \gamma_{1}}}}, 0\right)=\left(\frac{\beta_{2} \gamma_{1}-A_{1} A_{2}}{A_{1} B_{2}}, 0\right)=B_{0}
\end{aligned}
$$

and

$$
\begin{aligned}
\lim _{n \rightarrow \infty} & T^{2 n+1}(x, 0) \\
= & \lim _{n \rightarrow \infty}\left(0, \frac{\beta_{2}\left(\gamma_{1} \beta_{2}\right)^{n} x}{A_{2}\left[\left(A_{1} A_{2}\right)^{n}+A_{1} B_{2}\left(A_{1} A_{2}\right)^{n-1} x+\cdots+A_{1} B_{2}\left(\gamma_{1} \beta_{2}\right)^{n-1} x\right]+B_{2}\left(\gamma_{1} \beta_{2}\right)^{n} x}\right) \\
= & \lim _{n \rightarrow \infty}\left(0, \frac{\beta_{2} x}{A_{2}\left(\left(\frac{A_{1} A_{2}}{\beta_{2} \gamma_{1}}\right)^{n}+x\left(\frac{A_{1} B_{2}}{\beta_{2} \gamma_{1}}\right) \frac{1-\left(\frac{A_{1} A_{2}}{\beta_{2} \gamma_{1}}\right)^{n}}{1-\frac{A_{1} A_{2}}{\beta_{2} \gamma_{1}}}\right)+B_{2} x}\right)=\left(0, \frac{\beta_{2} \gamma_{1}-A_{1} A_{2}}{\gamma_{1} B_{2}}\right)=A_{0} .
\end{aligned}
$$

Lemma 8 The map $T^{2}$ associated to System (2) satisfies the following:

$$
T^{2}(x, y)=(\bar{x}, \bar{y}) \quad \text { only for }(x, y)=(\bar{x}, \bar{y})
$$

Proof Since $T^{2}$ is injective, then $T^{2}(x, y)=(\bar{x}, \bar{y})=T^{2}(\bar{x}, \bar{y}) \Rightarrow(x, y)=(\bar{x}, \bar{y})$.

\section{Proof of Theorem 1}

Case $1 \beta_{2} \gamma_{1} \leq A_{1} A_{2}$

Equilibrium $E_{0}$ is unique (see Lemma 1), and by Lemma 5, every solution of System (2) belongs to

$$
B=\left[0, \frac{\beta_{2} \gamma_{1}}{A_{1} B_{2}}\right] \times\left[0, \frac{\beta_{2}}{B_{2}}\right],
$$

which is an invariant box. In view of Corollary 1 and Theorem 2, every solution converges to minimal period-two solutions or $E_{0}$. System (2) has no minimal period-two solutions (Lemma 2). So, every solution of System (2) converges to $E_{0}$.

Case $2 \beta_{2} \gamma_{1}-A_{1} A_{2}>-B_{2}\left[A_{1}^{2}+\gamma_{1}\left(A_{2}-A_{1} B_{2}\right)\right]$ and $\beta_{2} \gamma_{1}-A_{1} A_{2}>0$

By Lemmas 1, 2, 4 and Theorems 6 and 7, there exist two equilibrium points: $E_{0}$ which is a repeller and $E_{+}$which is a saddle point, and minimal period-two solutions $A_{0}$ and $B_{0}$ which are locally asymptotically stable. Clearly $T^{2}$ is strongly competitive and it is easy to check that the points $A_{0}$ and $B_{0}$ are locally asymptotically stable for $T^{2}$ as well. System (2) can be decomposed into the system of the even-indexed and odd-indexed terms as follows:

$$
\left\{\begin{array}{l}
x_{2 n+1}=\frac{\gamma_{1} y_{2 n}}{A_{1}+x_{2 n}}, \\
x_{2 n}=\frac{\gamma_{1} y_{2 n-1}}{A_{1}+x_{2 n-1}}, \\
y_{2 n+1}=\frac{\beta_{2} x_{2 n}}{A_{2}+B_{2} x_{2 n}+y_{2 n}}, \\
y_{2 n}=\frac{\beta_{2} x_{2 n-1}}{A_{2}+B_{2} x_{2 n-1}+y_{2 n-1}}, \quad n=1,2, \ldots
\end{array}\right.
$$


The existence of the set $\mathcal{C}$ with the stated properties follows from Lemmas 6, 2, 7, 8, Corollary 1 , Theorems 4 and 5.

Case $30<\beta_{2} \gamma_{1}-A_{1} A_{2}=-B_{2}\left[A_{1}^{2}+\gamma_{1}\left(A_{2}-A_{1} B_{2}\right)\right]$

Cases (i) and (ii) from (c) in Theorem 1 are the consequence of Lemmas 1, 2, 4 and Theorems 6 and 7 .

Since $T^{2}$ is strongly competitive and points $A_{x}$ and $B_{x}$, for all $x \in\left[0, \frac{\beta_{2} \gamma_{1}-A_{1} A_{2}}{A_{1} B_{2}}\right)$, are nonhyperbolic points of the map $T^{2}$, by Lemmas $1,6,2,3,7$, Corollary 1 , Theorems 2, 5, 6 and 7, it follows that all conditions of Theorem 4 are satisfied for the map $T^{2}$ with $\mathcal{R}=[0, \infty) \times[0, \infty)$. By Lemma 7 , it is clear that

$$
\mathcal{C}_{A_{0}}=\{(x, y): x=0, y>0\} \quad \text { and } \quad \mathcal{C}_{B_{0}}=\{(x, y): x>0, y=0\}
$$

Case $40<\beta_{2} \gamma_{1}-A_{1} A_{2}<-B_{2}\left[A_{1}^{2}+\gamma_{1}\left(A_{2}-A_{1} B_{2}\right)\right]$

Lemma 2 implies that System (2) has minimal period-two solutions (14). Furthermore, Corollary 1 and Theorem 2 imply that all solutions of System (2) converge to an equilibrium or minimal period-two solutions, and since, by Theorem $6, E_{0}$ is a repeller, all solutions converge to $E_{+}$(which is, in view of Theorem 7 , locally asymptotically stable) or minimal period-two solutions (14). The points $A_{0}$ and $B_{0}$ are saddle points of the strongly competitive map $T^{2}$; and by Lemma 7, the stable manifold of $A_{0}$ (under $T^{2}$ ) is

$$
\mathcal{B}\left(A_{0}\right)=\{(x, y): x=0, y>0\}
$$

and the stable manifold of $B_{0}$ (under $T^{2}$ ) is

$$
\mathcal{B}\left(B_{0}\right)=\{(x, y): x>0, y=0\}
$$

and each of these stable manifolds is unique. This implies that the basin of attraction of the equilibrium point $E_{+}$is the set

$$
\mathcal{B}\left(E_{+}\right)=\{(x, y): x>0, y>0\},
$$

and Lemma 7 completes the conclusion (d) of Theorem 1.

\section{Competing interests}

The authors declare that they have no competing interests.

\section{Authors' contributions}

Both authors contributed to each part of this study equally and read and approved the final version of the manuscript.

\section{Acknowledgements}

The authors are very grateful to Professor M.R.S. Kulenović for his valuable suggestions. They thank also the referees for their useful comments.

Received: 20 March 2012 Accepted: 23 August 2012 Published: 4 September 2012

\section{References}

1. Camouzis, E, Kulenović, MRS, Ladas, G, Merino, O: Rational systems in the plane - open problems and conjectures. J. Differ. Equ. Appl. 15, 303-323 (2009)

2. Clark, D, Kulenović, MRS: On a coupled system of rational difference equations. Comput. Math. Appl. 43, 849-867 (2002)

3. Clark, CA, Kulenović, MRS, Selgrade, JF: On a system of rational difference equations. J. Differ. Equ. Appl. 11, 565-580 (2005) 
4. Hirsch, M, Smith, H: Monotone Dynamical Systems. In: Handbook of Differential Equations. Ordinary Differential Equations, vol. 2, pp. 239-357. Elsevier, Amsterdam (2005)

5. Kulenović, MRS, Ladas, G: Dynamics of Second Order Rational Difference Equations with Open Problems and Conjectures. Chapman and Hall/CRC, Boca Raton (2001)

6. Kulenović, MRS, Merino, O: Discrete Dynamical Systems and Difference Equations with Mathematica. Chapman and Hall/CRC, Boca Raton (2002)

7. Kulenović, MRS, Nurkanović, M: Asymptotic behavior of a system of linear fractional difference equations. J. Inequal. Appl. 2005, 127-144 (2005)

8. Kulenović, MRS, Nurkanović, M: Asymptotic behavior of a competitive system of linear fractional difference equations. Adv. Differ. Equ. 2006, 1-13 (2006)

9. Brett, A, Garić-Demirović, M, Kulenović, MRS, Nurkanović, M: Global behavior of two competitive rational systems of difference equations in the plane. Commun. Appl. Nonlinear Anal. 16, 1-18 (2009)

10. Garic-Demirović, M, Kulenović, MRS, Nurkanović, M: Global behavior of four competitive rational systems of difference equations in the plane. Discrete Dyn. Nat. Soc. 2009, Article ID 153058 (2009)

11. Smith, HL: Planar competitive and cooperative difference equations. J. Differ. Equ. Appl. 3, 335-357 (1998)

12. Smith, HL: The discrete dynamics of monotonically decomposable maps. J. Math. Biol. 53, 747-758 (2006)

13. Kalabušsić, S, Kulenović, MRS: Dynamics of certain anti-competitive systems of rational difference equations in the plane. J. Differ. Equ. Appl. 17, 1599-1615 (2011)

14. Garic-Demirović, M, Nurkanović, M: Dynamics of an anti-competitive two dimensional rational system of difference equations. Sarajevo J. Math. 7(19), 39-56 (2011)

15. Kalabušić, S, Kulenović, MRS, Pilav, E: Global dynamics of anti-competitive systems in the plane (submitted)

16. Kulenović, MRS, Merino, O: Competitive-exclusion versus competitive-coexistence for systems in the plane. Discrete Contin. Dyn. Syst., Ser. B 6, 1141-1156 (2006)

17. Kulenović, MRS, Merino, O: Global bifurcation for discrete competitive systems in the plane. Discrete Contin. Dyn Syst., Ser. B 12, 133-149 (2009)

18. Robinson, C: Stability, Symbolic Dynamics, and Chaos. CRC Press, Boca Raton (1995)

19. Kulenović, MRS, Merino, O: Invariant manifolds for competitive discrete systems in the plane. Int. J. Bifurc. Chaos 20, 2471-2486 (2010)

20. Clark, D, Kulenović, MRS, Selgrade, JF: Global asymptotic behavior of a two dimensional difference equation modelling competition. Nonlinear Anal. TMA 52, 1765-1776 (2003)

21. Kulenović, MRS, Merino, O: A global attractivity result for maps with invariant boxes. Discrete Contin. Dyn. Syst., Ser. B 6, 97-110 (2006)

doi:10.1186/1687-1847-2012-153

Cite this article as: Moranjkić and Nurkanović: Basins of attraction of certain rational anti-competitive system of difference equations in the plane. Advances in Difference Equations 2012 2012:153.

\section{Submit your manuscript to a SpringerOpen ${ }^{\circ}$ journal and benefit from:}

- Convenient online submission

- Rigorous peer review

- Immediate publication on acceptance

- Open access: articles freely available online

- High visibility within the field

- Retaining the copyright to your article 